\title{
El rol de las mujeres en la bioeconomía: El caso del vainillo
}

\section{The role of women in bioeconomy: The case of vainillo}

\author{
Luz María Castro 1 https://orcid.org/0000-0003-2867-0252, \\ Diana Encalada Jumbo ${ }^{1}$ https://orcid.org/0000-0001-6462-1277
}

${ }^{1}$ Departamento de Economía, Universidad Técnica Particular de Loja, Loja, Ecuador lmcastro4dutpl.edu.ec, dencaladaleutpl.edu.ec

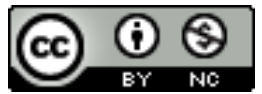

Esta obra está bajo una licencia internacional Creative Commons Atribución-NoComercial 4.0.
Enviado:

Aceptado:

Publicado:

\section{Resumen}

La bioeconomía se está posicionando como una opción viable para la gestión sostenible de los recursos naturales en el Ecuador. Las alternativas que promueve el Estado y otros organismos requieren considerar el rol de las mujeres como usuarias primordiales del bosque. Con la finalidad analizar la relación mujer- bosque, se estudió el caso de la Caesalpinia spinosa (Molina) Kuntze conocida como "vainillo", que es una especie nativa de los Andes con gran potencial en las industrias textil y alimenticia. Para conocer las características de los recolectores de vainillo, se aplicó una encuesta a 125 familias en la provincia de Loja. Según los resultados obtenidos, quienes se dedican a esta actividad son en su mayoría mujeres y personas mayores. La recolección se realiza entre mayo y noviembre, período que coincide con la estación seca y que se caracteriza por la migración masculina. Esta actividad genera en promedio USD 100 dólares al año, que complementa los ingresos por agricultura. El 95\% de recolectores depende de los bosques naturales, por lo que el cuidado y acceso a este recurso es esencial. Las iniciativas para promover la producción del vainillo deben aplicar estrategias con enfoque de género, para empoderar a las recolectoras en emprendimientos.

Palabras clave: bioeconomía, mujer, vainillo.

\begin{abstract}
Bioeconomy is positioning itself as a viable option for sustainable management of natural resources in Ecuador. Most of the alternatives promoted by the State and other organizations require considering the role of women as primary users of the forest. To analyse the relationship women-forest, the case of the Caesalpinia spinosa (Molina) Kuntze known as "vainillo", a native species of the Andes with great potential in the textile and food industries, was studied.
\end{abstract}

Sumario: Introducción, Productos forestales y su potencial bioeconómico, Metodología, Análisis de resultados, Discusión y Conclusiones.

Como citar: Castro, L. M. \& Encalada, D. (2021). El rol de las mujeres en la bioeconomía: El caso del vainillo. Revista Tecnológica - Espol, 33(3), 126-138.

http://www.rte.espol.edu.ec/index.php/tecnologica/article/view/875 
To learn about the socioeconomic characteristics of vainillo collectors, a survey was applied to 125 families in the province of Loja. According to the results, most of the people involved in this activity are women and older people. Harvesting takes place between May and November, a period that coincides with the dry season and is characterized by male migration. This activity generates an average of US $\$ 100$ per year, which complements income from agriculture. Ninety-five percent of harvesters depend on natural forests, so care and access to this resource are essential. Initiatives to promote vainillo should apply gender-focused strategies to empower women harvesters.

Keywords: bioeconomy, women, vainillo.

\section{Introducción}

Cuarenta años de investigación sobre género ponen de manifiesto que es una categoría importante a tener en cuenta en el diseño de políticas y prácticas ambientales. En la actualidad, existen fondos nacionales e internacionales que priorizan la promoción del enfoque de género en las organizaciones ambientales y de desarrollo para afianzar los criterios de sostenibilidad (Arora-Jonsson 2014). La investigación de género abarca un amplio espectro de ámbitos centrados en género y medio ambiente desde la década de 1970, en los cuáles se expone la necesidad de incluir estas variables cómo una categoría esencial de análisis en temas relacionados a la gobernanza ambiental y la gestión de recursos, y en las políticas locales, nacionales e internacionales (Hawkins \& Ojeda, 2011).

El trabajo de académicos que integran género y ciencias sociales con gestión ambiental ha cambiado drásticamente a lo largo de los años (Ramdas 2009). Se ha desmitificado el rol de los productos forestales en el valor del bosque, reconociendo el rol de los productos forestales no maderables y de los servicios ambientales en favor de la sociedad. Así, el enfoque que consideraba a los bosques y a la silvicultura como un dominio principalmente masculino también ha cambiado (Arora-Jonsson 2014). Tener en consideración el hecho de que los diferentes grupos sociales tienen diversas formas de relacionarse con el medio ambiente se convirtió en una pauta para varias iniciativas de política y gestión de los recursos del bosque. Sin embargo, en la mayoría de los países en desarrollo, aún se considera que satisfacer las necesidades de alimentos es responsabilidad de las mujeres, mientras los hombres son los principales recolectores de productos de alto valor, como la madera que requieren trabajo físico (Aswaf et al. 2013).

Parte de esta evolución en la gestión de recursos naturales, ha impulsado una mayor inclusión de las mujeres en los ámbitos de manejo forestal sostenible. Sin embargo, se observa que la participación de las mujeres se ve comprometida por aspectos culturales, sociales y económicos que afectan su integración a la producción y comercialización de productos forestales maderables y no maderables (Coleman y Mwangi 2013). Los roles de género se pueden ver en dos niveles diferentes: entre miembros femeninos y masculinos dentro de un hogar y entre hogares encabezados por hombres o mujeres. Dentro de los hogares, los hombres y las mujeres a menudo tienen diferentes roles y responsabilidades con respecto a la recolección, procesamiento y comercialización de productos forestales (Aswaf et al. 2013).

Es necesario, por tanto, que los programas que fomentan el manejo de recursos naturales con alto potencial para la bioeconomía en el Ecuador consideren el rol de la mujer en la gestión sostenible del recurso, cómo una vía para transformar las relaciones de poder y aliviar la pobreza en zonas rurales deprimidas. Parte de estos desafíos implica comprender los patrones de comportamiento y toma de decisiones de hombres y mujeres, que de acuerdo a Agarwal 
(2010) pueden seguir trayectorias diferenciadas, siendo los intereses de los hombres más ocasionales y a largo plazo, y los de las mujeres más cotidianos.

La bioeconomía ofrece oportunidades de integración de las comunidades rurales mediante la comercialización y transformación de los productos del bosque. Sin embargo, es primordial que se involucre a todos los actores relacionados a la cadena de valor. La evidencia sugiere que muchos programas basados en el mercado a menudo ignoran las preferencias de las mujeres y la comprensión de sus medios de vida (Arora-Jonsson 2014).

\section{Productos forestales y su potencial bioeconómico}

El concepto de un nuevo modelo económico es indispensable en medio de los esfuerzos globales para reducir el desperdicio, utilizar los recursos de manera sostenible y poner la naturaleza en primer lugar. Asegurar la sostenibilidad en el tiempo significa mantener un equilibrio dinámico entre una población humana en crecimiento y sus demandas, las capacidades cambiantes del entorno físico para absorber los desechos de la actividad humana, las posibilidades cambiantes abiertas por los nuevos conocimientos y cambios tecnológicos y los valores, aspiraciones e instituciones que canalizan el comportamiento humano (Aguilar et al. 2019).

La bioeconomía es un sector económico transversal emergente que produce, transforma y utiliza materiales y productos de base biológica, esto incluye directa e indirectamente sectores de base biológica como la silvicultura o la agricultura, y se encuentra en el centro de las estrategias económicas sostenibles de los países de todo el mundo (Biber-Freudenberger et al. 2020). A pesar de la diversidad en los puntos de partida de los países, que se deben a las diferencias en el desarrollo económico e industrial, el clima, las prácticas agrícolas, se observa que existe un conjunto común de iniciativas políticas que incluye la promoción de la innovación, el desarrollo de infraestructura y capacidades, mejora de la comercialización y armonización del marco regulatorio, especificaciones y estándares.

Ecuador al ser un país rico en recursos biológicos, tiene un gran potencial para integrarse en este modelo bioeconómico que busca el óptimo uso de recursos naturales a través de procesos tecnológicos. Uno de los primeros pasos a nivel político ha sido la firma del Pacto Nacional por la Bioeconomía Sostenible. Mediante este acuerdo interinstitucional se busca promover a la bioeconomía como una herramienta que permitirá enfrentar los desafíos en torno al cambio climático, la equidad e inclusión social, la protección de los recursos de origen biológico, la transición hacia una economía de conocimiento y una vía para promover la reactivación económica del país (MAE 2020).

Para dar ese paso, es necesario identificar productos con alto potencial para la industria y su cadena de valor, que incluye a los productores primarios. Así, el vainillo Caesalpinia spinosa (Molina) Kuntze, también conocido como tara, es uno de los productos mas prometedores en la industria alimenticia y textil a nivel mundial. La goma de vainillo, que se deriva de las semillas de este árbol, tiene un uso común como aditivo alimentario natural (Larrea 2011). Su costo es mayor que el del almidón o la gelatina, pero se necesita menor cantidad y la goma no afecta el sabor ni el color como otras gomas que provienen de exudados de especies forestales (Barriga, 2008). Además de goma (endospermo), las vainas contienen taninos, compuestos en un 53\% de ácido gálico, que se utilizan en la industria del cuero, donde otros taninos vegetales compiten con el del vainillo, como el de la Schinopsis spp, Castanea sativa o Acacia mollisima. La producción de vainillo tiene una ventaja comparativa frente a 
estas especies, en las que para extraer el tanino es necesario sacrificar los árboles, ya que se encuentra en la corteza y/o madera del árbol (Shangay-Tucto y Duponnois 2018).

El vainillo es una especie vegetal nativa de los Andes usada en forma habitual por las comunidades, y que en años recientes se comercializa para la industria química. Es un producto natural alternativo de exportación no tradicional, de alta rentabilidad económica frente a otros cultivos andinos (OSEC 2012). En la última década, la demanda del producto ha aumentado debido al crecimiento de mercados internacionales incluido Oriente Medio y Asia. La creciente conciencia sobre los productos alimenticios y cosméticos de origen natural muestra un crecimiento sustancial para los derivados del vainillo en los próximos 10 años (Romero 2019).

El crecimiento del comercio del vainillo ha abierto un nuevo nicho de mercado en la economía ecuatoriana y en la provincia de Loja, en donde el vainillo crece en forma natural. Las características ecológicas de la especie y su plasticidad han permitido que se distribuya en una amplia zona geográfica, particularmente en los valles secos interandinos. La mayor parte de la producción proviene de bosques nativos sin manejo y dispersos, lo que implica un mayor esfuerzo durante la época de cosecha, suponiendo un elevado costo de oportunidad frente a otras opciones cómo la agricultura y ganadería.

En áreas remotas donde se encuentran abundancia de la especie no se aprovecha por falta de mano disponible y los elevados costos de transporte que implica. En la zona de estudio, es común que las mujeres realicen la cosecha del vainillo como una actividad paralela a sus actividades diarias que incluyen la producción agrícola y cuidado del hogar. Durante el desplazamiento a la chacra las mujeres colectan las vainas de árboles que encuentran en zonas aledañas y van almacenando la producción para luego comercializarla.

El trabajo femenino es esencial para mejorar los procesos de recolección y ampliar la producción de vainillo en áreas silvestres no aprovechadas, e incluso en zonas agroforestales y plantaciones forestales. Sin embargo, existen limitaciones relacionadas a la independencia y autonomía económicas de las mujeres que limitan su empoderamiento en esta actividad productiva de gran potencial en la zona de estudio. En los últimos años ha habido mucho interés en el papel de la mujer en el manejo de recursos forestales. Particularmente en el sector forestal, la participación se ha visto como un camino hacia la mejora de la gobernanza, la promoción del uso y la gestión sostenibles, al mismo tiempo que se mejoran los beneficios y las oportunidades de los medios de vida (Coleman y Mwangi 2013).

Si la participación de las mujeres facilita el uso sostenible o mejora los medios de vida sigue siendo objeto de un intenso debate. Sin embargo, es evidente que cumplen un rol muy importante en la gestión de productos forestales no maderables (PFNM), que en muchas ocasiones no son del interés de sus pares masculinos. Heltberg et al. (2000) resalta el hecho de que las mujeres se dedican principalmente a la recolección de PFNM, mientras que los hombres participan en otras actividades generadoras de ingresos que incluyen la explotación forestal y la venta de mano de obra. Sin embargo, es evidente que los ingresos forestales, principalmente de los PFNM, son una fuente importante de ingresos tanto de subsistencia como en efectivo, especialmente para los grupos de bajos ingresos, ya que brinda la oportunidad de diversificar sus medios de vida (Dash et al. 2016).

Resulta de vital importancia identificar los factores que promueven la participación de las mujeres para impulsar el sector forestal con el fin de mejorar la participación de los grupos marginados en los procesos de toma de decisiones y que tendrán un gran impacto en el crecimiento de sectores asociados a la bioeconomía. La presente investigación tuvo como 
objetivos identificar las principales fuentes de ingresos de los hogares en el área de estudio, analizar la contribución relativa de los ingresos del vainillo al ingreso anual del hogar, y discutir el rol de la mujer en la promoción de actividades basadas en la bioeconomía, el manejo sostenible de recursos y la disminución de la pobreza en zonas rurales.

\section{Área de estudio}

\section{Metodología}

El estudio para caracterizar las unidades productoras de vainillo se realizó en los cantones Paltas, Gonzanamá y Catamayo en la provincia de Loja porque juntos representan el $63 \%$ de la producción estimada de vainillo con fines de exportación en Ecuador (NCI, 2017) (Figura 1). La zona de estudio está ubicada en la cuenca del río Catamayo a una altitud media de $1385 \mathrm{msnm}$. La precipitación media anual es $953 \mathrm{~mm}$ y la temperatura media anual es de $18{ }^{\circ} \mathrm{C}$. Tiene una extensión aproximada de $2396 \mathrm{~km} 2$, caracterizados por un clima templado y seco, con dos estaciones bien marcadas verano (seco) e invierno (lluvioso), esta última de diciembre a abril. En 2019, el tamaño de la población se estimó en 34.863 habitantes, de los cuales aproximadamente el $80 \%$ vive en áreas rurales y el $84 \%$ son clasificados como pobres por necesidades básicas insatisfechas (INEC, 2019).

\section{Figura 1}

\section{Mapa de distribución de Tara en la provincia de Loja}

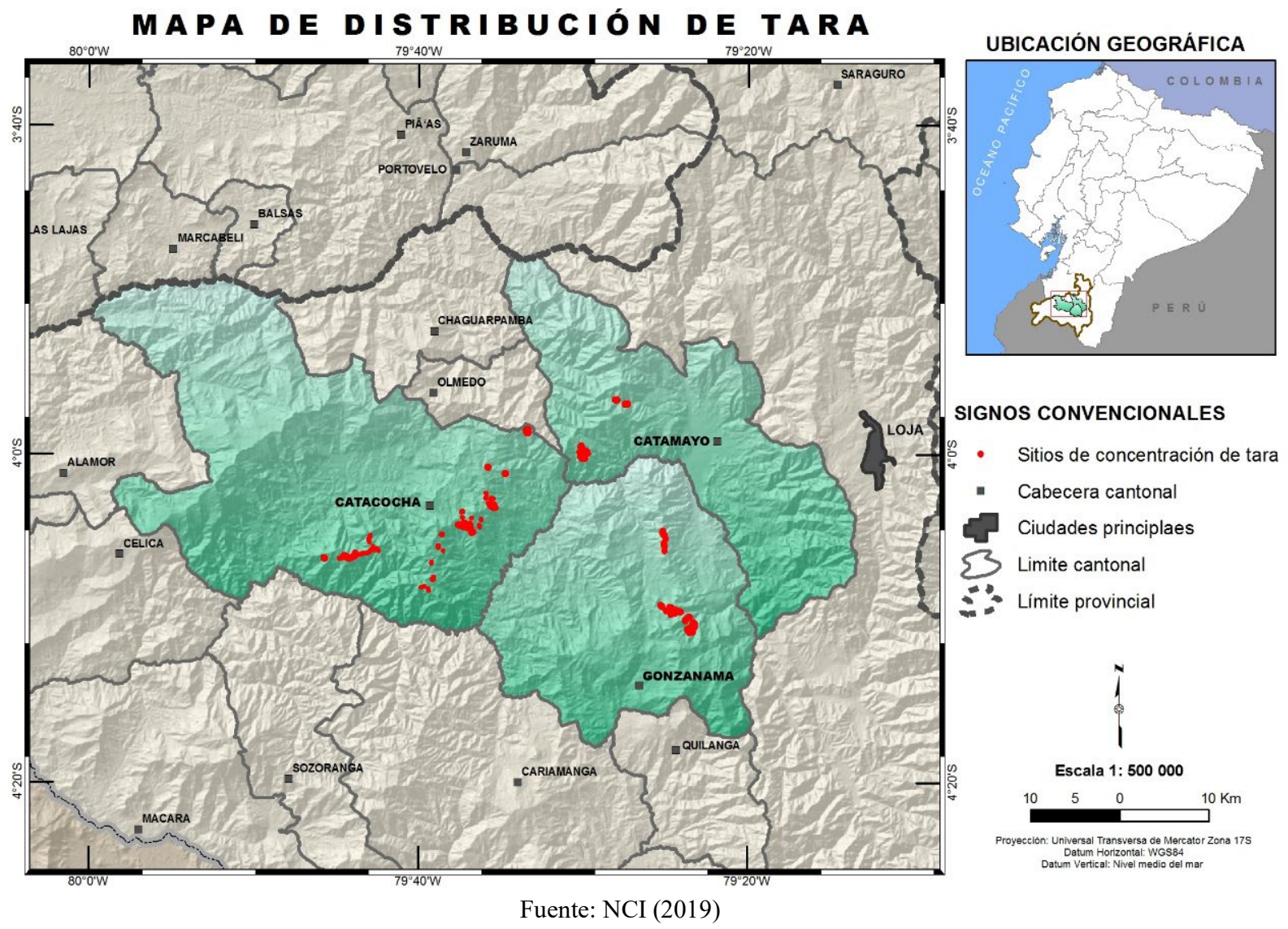

\section{Recolección y análisis de datos}

Para conocer las características socioeconómicas de los recolectores de vainillo se utilizó una encuesta semiestructurada. El cuestionario se centró en las características demográficas y socioeconómicas del hogar, los activos y las fuentes de ingresos, la 
participación de los diferentes miembros del hogar en las diferentes actividades generadoras de ingresos, como la agricultura, la ganadería, la extracción forestal maderera y no maderera; recolección y venta de vainillo. Se llevaron a cabo 125 encuestas a recolectores y productores en sus fincas, en el período de recolección comprendido entre enero y julio de 2019.

Para el procesamiento de datos se utilizaron los programas estadísticos Excel y Stata 14. Para el análisis descriptivo de resultados se calcularon estadísticos como la media, desviación estándar y coeficiente de variación y proporción de las características cualitativas y cuantitativas con las que fue construido el instrumento. Los resultados se agruparon en aspectos socioeconómicos y demográficos de los recolectores de vainillo, así como en la producción agrícola, ganadera y forestal maderable y no maderable para caracterizar los medios de vida de la población.

\section{Método de valoración del ingreso familiar}

Se identificaron todos los productos agrícolas producidos por un hogar, se estimaron sus volúmenes y se multiplicaron por el precio del mercado local por unidad de volumen para llegar al ingreso total de la granja. Los ingresos agrícolas totales se añadieron a los ingresos forestales y a los ingresos que un hogar obtenía de las actividades agrícolas y no agrícolas para proporcionar la estimación del ingreso familiar Los principales costos de producción considerados se relacionan con los insumos y la mano de obra. Estos costos se calcularon y se dedujeron del ingreso total del hogar para estimar el ingreso neto del hogar. En consecuencia, el ingreso neto, por ejemplo, de la cosecha se calculó como la diferencia entre el ingreso total de todas las actividades de producción de cultivos y los costos de insumos incurridos para la producción (costos de fertilizantes inorgánicos, estiércol, semillas mejoradas y pesticidas, alquiler pagado por la tierra arrendada e intereses pagados por el capital agrícola prestado).

Los ingresos forestales se calcularon estimando el volumen total de productos forestales recolectados por un hogar y multiplicado por el precio de mercado por unidad de volumen. Los ingresos de las actividades agrícolas y no agrícolas incluyen los ingresos del empleo laboral ocasional, el empleo asalariado, el pequeño comercio, las remesas y diferentes formas de ayuda recibidas de las ONG o del gobierno.

\section{Características de los productores}

\section{Análisis de Resultados}

Los productores de vainillo se ubican en tres cantones de la provincia. El $67.44 \%$ de los productores estan ubicados en el cantón Gonzanamá, el 37.78\% pertenecen al cantón Paltas y solo el $0.78 \%$ corresponden al cantón Catamayo. A nivel de parroquia, la mayor población de productores y recolectores se localiza en Nambacola del cantón Gonzanamá.

En cuanto a las características de las personas encuestadas, es posible indicar que la producción de vainillo es mayoritariamente femenina. El 65\% de las personas dedicadas a las labores de recolección son mujeres, mientras que el $35 \%$ son hombres. Las mujeres tienden a participar en mayor medida en la recolección de vainillo, debido a que esta actividad representa una oportunidad para incrementar sus ingresos, al tiempo que pueden permanecer en las labores agrícolas, pecuarias y del hogar que realizan de manera cotidiana. La edad de los encuestados va desde los 24 años a los 93 años, sin embargo, la mayoría de la población se localiza en una edad comprendida entre los 50 a los 60 años, con una media de 58 años, lo que indica que la producción está en manos de adultos mayores.

El tamaño medio del hogar es relativamente pequeño con 3,48 personas. Aproximadamente, una tercera parte de los hogares (29\%) están compuestos por dos miembros. 
Lo que antecede es debido a que varios miembros de la familia han migrado, principalmente por trabajo, a otras ciudades de Ecuador y/o a otros países; quedando, en muchos casos, únicamente el padre y la madre mayores, y, en otros casos, también los niños $(32,8 \%)$.

En cuanto a educación, el $48.84 \%$ de los encuestados han culminado la primaria, el $23.26 \%$ han culminado la secundaria, el $13.18 \%$ han iniciado la educación primaria pero no la han culminado, el 7.75\% han iniciado la educación secundaria sin haberla culminado, el 3.10\% no poseen ningún nivel de instrucción, el $2.33 \%$ posee un título de tercer nivel, mientras que el $1.55 \%$ no ha culminado la educación superior.

\section{Medios de vida}

El 52\% de encuestados realiza dos actividades económicas, mientras que el $48 \%$ tiene una única fuente de ingreso para el hogar. Del grupo de encuestados que tienen dos actividades económicas el $21 \%$ son hombres y el $79 \%$ son mujeres. Existe una mayor tendencia por parte de la población femenina en la búsqueda de una segunda actividad económica, debido a que gran parte de las encuestadas se identifican como amas de casa, actividad por la cual no reciben remuneración.

El $72 \%$ de los jefes de hogar han identificado a la agricultura y ganadería como su actividad principal. La segunda actividad con mayor frecuencia es el trabajo en el hogar con el 14\%. Entre otras actividades económicas identificadas están el $9.30 \%$ que se considera trabajador privado, el $3.10 \%$ se encuentra jubilado, y el $0.78 \%$ es trabajador público. La mayor parte de los recolectores mantiene una producción agropecuaria de subsistencia, sin excedentes para el mercado lo que limita su capacidad de acumulación de capital y reinversión.

Las unidades productivas se caracterizan por producir en pequeña escala cultivos tradicionales como maíz y fréjol. Otros cultivos representativos son la arveja, el café, la zarandaja y el maní. Por lo general se produce en condiciones de temporal, en superficies pequeñas, utilizando mano de obra familiar y tecnología tradicional. El tamaño promedio de las fincas es de 4,19 ha. Solo el $25 \%$ de la tierra se aprovecha para fines productivos, esto puede atribuirse a la baja calidad del suelo en la zona; la falta de mano de obra, de riego y de recursos financieros, así como a la incertidumbre climática. En cuanto a la producción pecuaria, el $86 \%$ se dedican a la producción de ganado vacuno, porcino y aves de corral, así como de otros animales de granja como cuyes, equinos y caprinos. La mayor parte de la producción agrícola y pecuaria se destina al autoconsumo y los excedentes se comercializan en los mercados locales.

Con respecto a los productos forestales maderables y no maderables, el $20.03 \%$ aprovechan los productos forestales maderables, principalmente faique para leña y postes para cercar. Los dos únicos productos forestales no maderables que se extraen en este sector, estos productos son miel a la que se dedican el $0.78 \%$ y condurango (Marsdenia condurango) al que se dedican el $7.75 \%$.

El ingreso anual promedio del hogar incluida la producción agrícola, la ganadería, la recolección de PFNM como el vainillo y las actividades fuera de la finca es de USD 2.150 (46\% del salario básico unificado anual del país para el 2019, establecido en USD 4.728). El $88 \%$ de los hogares reporta ingresos inferiores al salario básico unificado anual y tan sólo el $2,4 \%$ reporta ingresos superiores al valor de la canasta básica familiar anual para la provincia de Loja (USD 8905 por año). Con los datos obtenidos, se estima que el $30 \%$ viven por debajo de la línea de pobreza del país, establecida para el 2019 en USD 1.020/año (INEC, 2019). La 
mayor parte de los ingresos se destina a cubrir gastos de alimentación (62\%) y con la diferencia se cubre los gastos de vivienda (8\%), transporte $(9 \%)$ salud $(8 \%)$ y educación $(13 \%)$.

\section{Producción de vainillo}

El vainillo se recolecta durante mayo a noviembre, período que coincide con la estación seca en esta zona. El 93\% de los encuestados realizan la recolección una vez al año, el 6\% de los recolectores recolecta hasta 2 veces por año y solo el $0.8 \%$ recolecta 3 veces por año. La cantidad de vainillo recolectada varía dependiendo del tipo de manejo. Aquellas personas que cosechan más de una vez al año son quienes realizan algún tipo de manejo forestal, como la poda o el riego.

La cosecha de vainillo es una actividad que se da mayormente en zonas silvestres. Las áreas aprovechadas son mayormente de tipo comunitario $(36,25 \mathrm{ha})$, mientras que las áreas privadas suman un total del 24,76 ha, el área aprovechada de terreno sin pago es de 9 ha. Alrededor del 30\% de los recolectores aplican técnicas de manejo en los bosques de vainillo de su propiedad, como la poda y aclareo/raleo de árboles.

Según indican los recolectores, el tiempo invertido para la cosecha depende de distancia hacia los sitios de recolección y la dispersión de los árboles. En el caso de las mujeres es una actividad paralela a la agricultura, muchas de ellas manifiestan que recogen vainillo en su recorrido hacia la huerta. En cuanto a la dispersión de los árboles, la media de tiempo invertido en la recolección en zonas donde los árboles se encuentran juntos es 4 horas por saco. Cuando los árboles se encuentran dispersos la media de tiempo invertido en la recolección es de 7 horas por saco. Además, de acuerdo a lo indicado por los recolectores y observación directa, el tiempo invertido depende de la cantidad de fruto del árbol, la técnica de recolección y la edad del recolector.

El promedio de cosecha es de 5 quintales por recolector. El quintal de vainillo se vende a un promedio de USD 17.8 dólares. La media del ingreso del vainillo es de USD 92 por recolector. Los costos que se consideran en la recolección son los de transporte, los cuales tienen una media de USD 0.90, obteniendo un beneficio promedio el USD 87.5. Es preocupante que los recolectores no valoran su mano de obra en la cuantificación del beneficio, ya que en promedio si los árboles están juntos cada productor emplearía medio jornal por saco, mientras que si están dispersos debería invertir un jornal completo por saco. Los jornales se valoran en la zona de estudio en USD15 dólares.

Entre las técnicas utilizadas, el $44 \%$ de personas recolectan el vainillo del piso, el $29 \%$ combinan la recolección del piso y el golpe de las ramas de los árboles, mientras que el 18\% recolectan del piso y sacudiendo el tronco. Como puede observarse no existe aplicación de ningún tipo de técnicas o herramientas que faciliten la recolección en forma eficiente y que permita una mejor calidad de las vainas. Es necesario que promuevan prácticas de cosecha que sean óptimas desde el enfoque de productividad y sostenibilidad. En cuanto a las actividades de manejo silvicultural los resultados indican un trabajo muy incipiente. Solo el $29 \%$ realizan un cuidado de las plantas, y realizan actividades de manejo como poda (35\%), raleo $(30 \%)$, enriquecimiento $(2.7 \%)$ y riego $(2.7 \%)$.

El 74\% de los hogares recolectores recibe entre USD 0 y 100 dólares anuales por la venta del vainillo, el 14\% entre USD 101 y 200 , el $8 \%$ entre USD 201 y 300 , el 4\% entre USD 301 y 400 y sólo el $0.8 \%$ más de USD 400 dólares. En promedio, la recaudación anual de vainillo (USD 91) representa el 4,23\% del ingreso anual total del hogar. 


\section{Cadena de valor}

La cadena de valor del vainillo está poco desarrollada aún, cuenta con un limitado número de actores, por lo que son fácilmente reconocibles los roles que desempeñan cada uno de ellos, así como su beneficio dentro de la cadena, como se muestra en la Figura 2. Los recolectores representan el $90 \%$ de los productores y su rendimiento por ciclo productivo alcanza los 5 sacos, siendo menor que el rendimiento de los productores que realizan actividades de reforestación que alcanzan los 8 sacos.

La producción y recolección del vainillo es aún una actividad poco explotada en la zona de estudio, al tratarse de un mercado local relativamente nuevo. El apoyo instituciones públicas como el GAD-Loja y el MAG, así como de organizaciones no gubernamentales como Naturaleza y Cultura Internacional y BOS + han sido clave para promover el aprovechamiento del vainillo y la reforestación de áreas para futuro aprovechamiento. Estas instituciones han apoyado con capacitaciones en temas relacionados a repoblación y manejo forestal. El trabajo en las técnicas de recolección ha recibido menor atención, y se observa que los productores utilizan procedimientos rudimentarios que no optimizan los recursos humanos disponibles.

Pese a los esfuerzos realizados, los productores recolectores/productores de vainillo aun consideran necesario fortalecer la capacitación y asistencia técnica para incrementar los volúmenes de producción y recolección del vainillo.

Existe demanda nacional e internacional insatisfecha, la cuál debería ser el mayor incentivo para impulsar la producción del cultivo de vainillo, ya que la proyección de la nueva industria de la curtiembre implica el uso de sustitutos vegetales; por ello hay y habrá cada vez en mayor número demandantes de insumos de guarango para las tenerías y curtiembres de todo el país. Lo mismo ocurre en el extranjero, en donde el auge de la demanda del polvo de guarango, para la industria de la curtiembre, se debe a la prohibición de la Unión Europea a partir del 2006, del uso de compuestos como el plomo, cadmio y cromo para el curtido de los cueros (Romero 2019).

\section{Figura 2}

\section{Cadena de valor del vainillo}
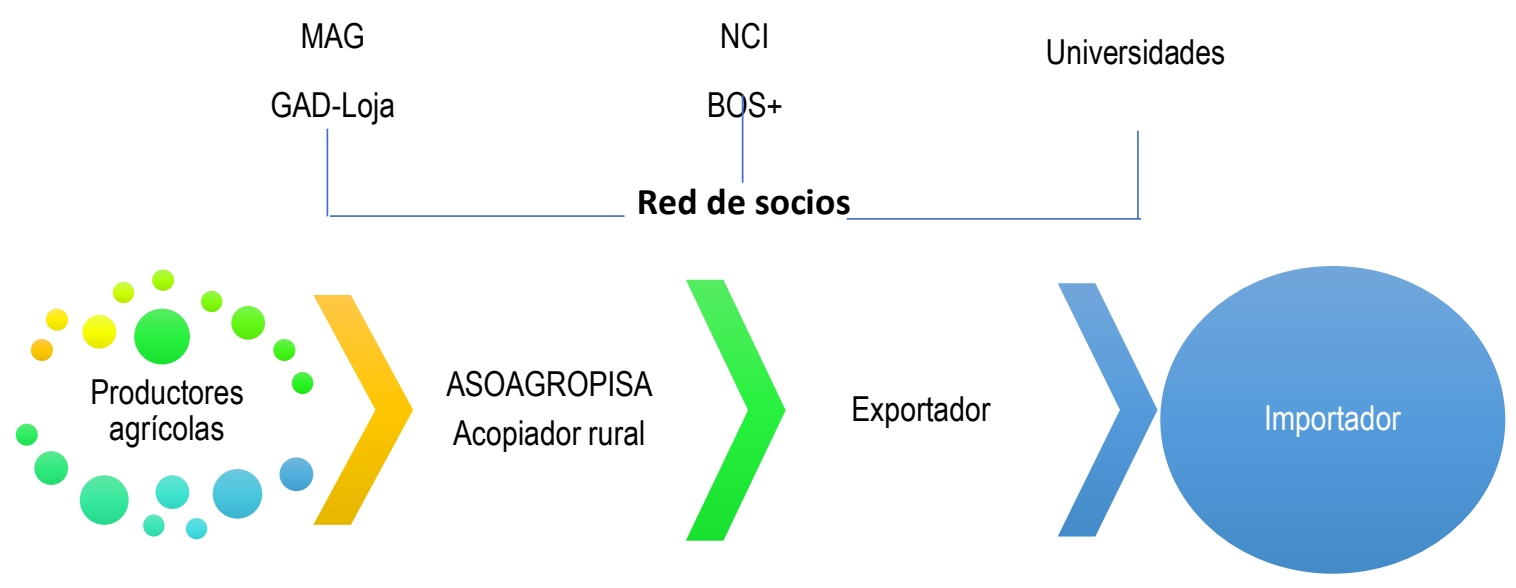

Influencias externas

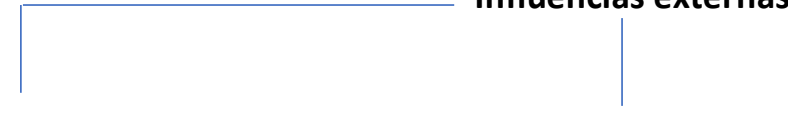

Cambio climático Acceso a crédito

Política de apoyo a emprendimientos forestales 


\section{Discusión}

En los últimos años, la investigación sobre el papel de los ingresos relacionados con los bosques en los medios de vida rurales ha ido ganando impulso. Existe un conjunto de estudios de casos en todo el mundo que investigan las interacciones entre los bosques y los medios de vida y encuentran que las contribuciones a los ingresos de los bosques oscilan entre el $6 \%$ y el 45\% (Dash et al 2016). En el caso del vainillo, en la actualidad representa menos del 5\% del ingreso familiar, por lo que es necesario promover entre los pobladores de la zona la recolección del fruto que regularmente se pierde en el árbol.

A pesar que varios estudios han encontrado que los jóvenes pueden ser más dependientes de los productos forestales que los ancianos (Godoy y Contreras, 2001), en la zona quienes se dedican al comercio del vainillo tienen en promedio 58 años. En la actualidad los jóvenes no están particularmente interesados en el producto. Esto se puede deber al hecho de que los jóvenes prefieran opciones que no demanden mucha mano de obra. Sin embargo, entre quienes se dedican son los recolectores más jóvenes, tanto en Gonzanamá como en Paltas, quienes presentan una mayor disposición a invertir en plantaciones comerciales de vainillo a futuro. Este resultado es relevante, ya que ello garantizaría la sostenibilidad de la producción del vainillo.

Los estudios encuentran que la educación hace que la recolección de PFNM sea cada vez menos rentable debido a los mayores costos de oportunidad de la mano de obra (Dash et al. 2016). En la zona de estudio solo el 5\% cuenta con educación superior. No hay diferencias significativas en los niveles de educación entre hombres y mujeres, pero si entre los recolectores de Paltas y los de Gonzanamá; los primeros tienen, en promedio, 3,3 años de educación más que los segundos.

Es asombroso que muchas personas aún no perciban el potencial de la recolección de vainillo como estrategia de diversificación. Los PFNM son activos relativamente líquidos que pueden recolectarse fácilmente del bosque sin costo y venderse en el mercado local en respuesta a las fluctuaciones de precios, o pueden usarse para el consumo doméstico. Sin embargo, Ndoye y Kaimowitz (2000) sugieren que un buen acceso al mercado implica menores ingresos forestales, ya que las oportunidades de ingresos alternativos son mejores.

\section{Mujer y empoderamiento en la agricultura}

Una gran cantidad de estudios sobre el papel de la mujer en la agricultura han puesto de relieve las brechas de género en la propiedad de activos, la educación, el acceso al crédito y los servicios de extensión, lo que hace que las agricultoras sean menos productivas. Para apoyar a las mujeres agricultoras a través de medidas políticas, es esencial comprender la dinámica que impulsa la brecha de género (Sell y Minot 2018).

Muchos estudios sugieren que la brecha de género está relacionada en gran medida con cuestiones relacionadas con la participación y el empoderamiento de las mujeres. Identificar, examinar y comprender estos determinantes es un primer paso en la exploración de estrategias para reducir la desigualdad de género y promover la seguridad alimentaria y la reducción de la pobreza. Se ha descubierto que poseer recursos productivos fortalece la posición negociadora de la mujer en el hogar (Meier zu Selhausen, 2016).

Con base a lo expuesto es necesario ampliar la investigación sobre las brechas de género en la zona de estudio y las dinámicas de poder que ocurren. Debido a los procesos de migración estacional y también permanente, los patrones de poder han evidenciado cambios en las últimas décadas. Muchas madres en la zona de estudio manifiestan que, aunque usan la tierra no pueden 
tomar decisiones de inversión y endeudamiento, debido a que no poseen títulos de propiedad a su nombre. Esta situación limita su independencia en la toma de decisiones.

Empoderar a las mujeres y reducir las desigualdades de género son dos objetivos clave para las políticas de desarrollo. Aunque existe un renovado interés en el sector agrícola como motor de crecimiento y desarrollo y un mayor reconocimiento de la importancia de la mujer en la agricultura, sin herramientas para medir el impacto de las intervenciones agrícolas en el empoderamiento de las mujeres, es probable que los impactos de los programas en el empoderamiento reciban menos atención que otros resultados más mensurables (Alkire et al. 2013).

\section{Política e incentivos}

La política para promover la gestión forestal debe tener en cuenta a los principales usuarios del bosque y los tipos de productos de los que dependen, y debe ir acompañada de otras medidas de reducción de la pobreza para mejorar los resultados de conservación de los bosques, de lo contrario tendrá consecuencias negativas en los medios de vida locales, especialmente en los pobres y las mujeres, que dependen más del bosque (Aswaf et al. 2013).

La inclusión de las mujeres en los mercados ha aumentado sus opciones y su poder de negociación en la familia, sin embargo, también ha sido la causa de conflictos al introducirlas a un sistema sobre el que tienen poco control. Las políticas que abogan por soluciones basadas en el mercado han promovido la idea de que las subvenciones económicas y los programas de generación de ingresos o la participación de las mujeres en las cadenas de productos / valor resolverían todos los problemas de las mujeres, problemas que de hecho surgen de relaciones desiguales de poder (Arora-Jonsson 2014).

Agarwal (2010) reconoce que en ámbito de la ordenación forestal el hallazgo más significativo es la capacidad de las mujeres para hacer efectivas estas instituciones. A pesar de su falta de experiencia con las instituciones públicas, a todas luces los consejos dirigidos íntegramente por mujeres o con más mujeres demostraron ser mejores guardianes del bosque. A pesar de sus necesidades más urgentes de productos forestales, los consejos con más mujeres apoyaron reglas más estrictas para el uso de los bosques, sanciones más severas y una implementación más consistente. Esto nos alienta a pensar que involucrar a las mujeres en la gestión de recursos es beneficioso desde el punto de vista socioeconómico y también de manejo sostenible.

\section{Conclusiones}

La producción y recolección del vainillo es aún una actividad comercial de gran potencial para la bioeconomía de la zona de estudio debido a la creciente demanda mundial de este tipo de materias primas, amigables con el ambiente, para la producción de taninos y gomas, especialmente utilizados en las industrias textil y alimenticia. Sin embargo, se encuentra en una fase incipiente de producción y generación de riqueza. En general, la mayor parte de los recolectores y productores se localizan en Gonzanamá (69\%), son mujeres $(65 \%)$ y mayores de 50 de años (70\%), con bajos niveles de educación (7.5 años) e ingresos ( $88 \%$ gana menos del salario básico unificado mensual del 2019 de USD394), cuya principal actividad económica es la agricultura y para quienes la recolección y producción del vainillo es una actividad complementaria. En promedio, cada hogar recolecta $230 \mathrm{~kg}$ de vainillo/año, principalmente en bosque natural (95\%). Los ingresos provenientes de la venta del vainillo representan tan sólo el $4 \%$ de los ingresos anuales de los hogares. 
Aunque el aprovechamiento del vainillo en la zona tiene potencial, se requiere un fuerte componente de capacitación en procesos de manejo silvicultural, financiero y de liderazgo para que más familias se vinculen al sector y que aumenten su participación en el mercado local y nacional. También se requiere inversión en para el establecimiento y manejo de plantaciones a futuro y tecnificación de la postcosecha, para fortalecer la cadena de valor a nivel local.

El rol de la mujer es de gran importancia en la zona de estudio debido a la composición de los hogares y los patrones de migración existentes. Garantizar el empoderamiento de las mujeres y su incursión en actividades económicas basadas en PFNM tendrá un impacto en sus ingresos y en el bienestar de sus familias. Adicionalmente puede tener un impacto muy positivo en términos de manejo sostenible del recurso conforme a experiencias documentadas por académicos.

\section{Referencias}

Agarwal B. (2010) Gender and green governance: The political economy of women's presence within and beyond community forestry. Oxford University Press, Oxford

Aguilar, A., Twardowski, T., Wohlgemuth, R. (2019) Bioeconomy for Sustainable Development. Biotechnology Journal. https://doi.org/10.1002/biot.201800638

Alkire, S., Meinzen, R., Peterman, A., Quisumbing, A. R., Seymour, G., Vaz, A. (2013) The Women's Empowerment in Agriculture Index. OPHI Working Paper NO. 58. University of Oxford

Arora-Jonsson, S. (2014) Forty years of gender research and environmental policy: Where do we stand? Women's Studies International Forum Volume 47: 295-308

Asfaw, A., Lemenih, M., Kassa, H., Ewnetu, Z. (2013) Importance, determinants and gender dimensions of forest income in eastern highlands of Ethiopia: The case of communities around Jelo Afromontane forest. Forest Policy and Economics 28: Pages 1-7

Barriga, C. (2008). Cultivos y aprovechamiento de la vainillo Caesalpinia spinosa en la región andina (documento de trabajo). ECOBONA. Lima.

Biber-Freudenberger, L., Ergeneman, C., Förster, J. J., Dietz, T., Börner, D. (2020) Bioeconomy futures: Expectation patterns of scientists and practitioners on the sustainability of bio-based transformation. Sustainable development https://doi.org/10.1002/sd.2072

Coleman, E. A., Mwangi, E. (2013) Women's participation in forest management: A cross-country analysis. Global Environmental Change 23 (1): 193-205.

Dash, M., Behera, B., Rahut, D. B. (2016) Determinants of household collection of non-timber forest products (NTFPs) and alternative livelihood activities in Similipal Tiger Reserve, India. Forest Policy and Economics 73: 215-228

Godoy, R., Contreras M. (2001) A comparative study of education and tropical deforestation among lowland Bolivian Amerindians: forest values, environmental externality, and school subsidies. Econ. Dev. Cult. Chang., 49 (3): 555-574

INEC (2019). Instituto Nacional de Estadísticas y Censos. Obtenido de https://www.ecuadorencifras.gob.ec/institucional/home/

Hawkins, R., Ojeda, D. (2011) Gender and environment: Critical tradition and new challenges Environment and planning D, 29: 237-253

Heltberg, R., Arndt, T.C., Sekhar, N.U. (2000) Fuelwood consumption and forest degradation: a household model for domestic energy consumption in rural India. Land Econ.76 (2): 213-232 
Larrea, M. (2011) La vainillo, guarango o taya (Caesalpinia spinosa) en la Región Andina: criterios ambientales para la sustentabilidad de su aprovechamiento y manejo en Bolivia, Ecuador y Perú. Ecobona, Serie de Capacitación No. 5 Programa Regional Ecobona-Intercooper, Quito, Peru.

MAE (2020). Ecuador promueve la bioeconomía como una estrategia para el Desarrollo Sostenible. Boletín $\mathrm{N}^{\circ}$ 188, Quito.

Meier zu Selhausen, F. (2016) What determines women's participation in collective action? Evidence from a Western Ugandan coffee cooperative. Feminist Economics, 22 (1): 130-157

Naturaleza y Cultura Internacional. (2017). Vainillo: exportaron primeras 48 toneladas. Retrieved December 2 , 2018, from http://www.naturalezaycultura.org/spanish/htm/news/2017-08-Vainillo.htm

Ndoye, O., Kaimowitz, D. (2000) Macro-economics, markets and the humid forests of Cameroon. J. Mod. Afr. Stud., 38 (2): 225-253

Osec (2012). Market Brief for Tara Gum. Swiss Import Promotion Programme. Disponible en http://www.biotrade.org/congress/BackgroundDocs2/General\%20docs/Peru/Peru_Market-Brief-Taragum_SIPPO.pdf

Ramdas, S. R. (2009) Women, forest spaces and the law: Transgressing the boundaries Economic and Political Weekly 44: 65-73

Romero, I. (2019). Producción y Comercio de la Tara en Perú. Ministerio de Agricultura y Riego, Dirección General de Políticas Agrarias. Lima: Dirección de Estudios Económicos e Información Agraria. Obtenido de http://www.siicex.gob.pe/siicex/documentosportal/alertas/documento/doc/168510289radF57F7.pdf

Sell, M., Minot, N. (2018) What factors explain women's empowerment? Decision-making among small-scale farmers in Uganda. Women's Studies International Forum 71: 46-55

Shangay-Tucto, S., Dubonnois, (2018) Ecological characteristics of vainillo (Caesalpinia spinosa), a multipurpose legume tree of high ecological and commercial value. In: Agricultural Research Updates. Volume 22. Editors: P. Gorawala et al. Nova Science Publishers, Inc. 